\title{
Práticas e perspectivas da terapia ocupacional na inclusão escolar*
}

\section{Practice and perspectives of school-based occupational therapy}

\author{
Paula Tatiana Cardoso ${ }^{1}$, Thelma Simões Matsukura²
}

CARDOSO, P. T.; MATSUKURA, T. S. Práticas e perspectivas da terapia ocupacional na inclusão escolar. Rev. Ter. Ocup. Univ. São Paulo, v. 23, n. 1, p. 7-15, jan./abr. 2012.

RESUMO: O objetivo do estudo foi identificar e caracterizar as ações desenvolvidas pela Terapia Ocupacional no processo de inclusão escolar de crianças com necessidades educacionais especiais no estado de São Paulo. Foi realizada uma pesquisa de levantamento que contou com a participação de 127 terapeutas ocupacionais. Os dados coletados por meio de questionário foram submetidos a análises quantitativas e qualitativas e os principais resultados indicaram que os terapeutas ocupacionais envolvidos com a inclusão escolar: a) se relacionam com este processo principalmente a partir do atendimento em ambiente clínico; b) atuam na escola regular; c) têm a participação da família e de outros profissionais em sua atuação; d) identificam a falta de preparação dos professores e da escola como os maiores obstáculos para o seu trabalho e e) identificam a importância e a necessidade de ampliação da inserção da Terapia Ocupacional nas escolas regulares. O estudo identificou características representativas da atuação dos terapeutas ocupacionais do estado de São Paulo no processo de inclusão escolar e levantou indicadores para estudos futuros.

DESCRITORES: Criança com Deficiência/educação; Educação especial/tendências; Educação especial/recursos humanos; Terapia ocupacional.

\footnotetext{
* Este artigo apresenta resultados da dissertação de mestrado intitulada "Inclusão escolar de crianças com necessidades especiais: práticas e perspectivas de terapeutas ocupacionais”, desenvolvida no Programa de Pós-Graduação em Educação Especial, da Universidade Federal de São Carlos (PPGEE/UFSCar) e contou com o apoio da Coordenação de Aperfeiçoamento de Pessoal de Nível Superior - CAPES.

1. Docente do curso de Terapia Ocupacional da Universidade Federal do Triângulo Mineiro - UFTM. Mestre em Educação Especial pela Universidade Federal de São Carlos - PPGEE/UFSCar.

2. Professora Associada do Departamento de Terapia Ocupacional. Docente do Programa de Pós-Graduação em Terapia Ocupacional e do Programa de Pós-Graduação em Educação Especial da UFSCar.

Endereço para correspondência: Universidade Federal de São Carlos - Departamento de Terapia Ocupacional - LAD. Rodovia Washington Luís, km 235 - São Carlos, SP. CEP: 13565-905.
} 


\section{INTRODUÇÃO}

\section{Inclusão escolar e a Terapia Ocupacional}

inclusão social discutida sob a perspectiva
da educação traz a proposta da inclusão
escolar, um dos principais desafios da escola atualmente, uma vez que representa transformações paradigmáticas, legislativas e cotidianas de alto impacto na estrutura educacional.

No Brasil, as principais mudanças legislativas nesse campo ocorreram após a participação do país em encontros promovidos pela Organização Mundial da Saúde (OMS) para discutir a educação das pessoas com necessidades especiais $^{(1)}$. Neles foram firmados acordos de mobilização mundial para garantia do direito à educação comum para todos e o respeito à diversidade. Destaca-se a participação do país na “Conferência Mundial da Educação”, na Tailândia em 1990 e na "Conferência Mundial sobre Necessidades Educacionais Especiais: Acesso e Qualidade”, ocorrida na cidade de Salamanca na Espanha, no ano de 1994.

Desde então, foram desenvolvidos no Brasil projetos e leis para priorizar, regularizar e possibilitar a educação de qualquer criança, adolescente ou adulto no sistema regular de ensino, independente de suas limitações. Neste contexto um dos tópicos mais debatidos é o envolvimento de diferentes campos de conhecimento na elaboração e desenvolvimento das práticas inclusivas; considera-se que este envolvimento aconteceria através da contribuição de profissionais de diferentes áreas atuando no processo de inclusão (MENDES, 2002; SASSAKI, 2002).

Nesta direção, Pelosi (2006) aponta o terapeuta ocupacional como um profissional que estará presente em muitas das equipes de apoio à inclusão escolar e destaca que a capacidade de reconhecer a diversidade e a possibilidade de favorecer a funcionalidade dos indivíduos a partir de suas potencialidades faz deste um profissional qualificado para atuar como facilitador da inclusão.

A atuação da Terapia Ocupacional em processos educacionais não é recente, a intervenção em instituições que desenvolvem programas de educação especial vem acompanhando a implementação e consolidação da profissão no Brasil desde meados da década de 1960 (SOARES, 1991; BARTALOTTI, 2007).
A inserção dos terapeutas ocupacionais no processo de inclusão escolar parece ser uma conseqüência da comunicação da profissão com a educação especial e com os processos de inclusão social em geral. No entanto, o terapeuta ocupacional precisa rever conceitos e ações para se colocar com confiança e responsabilidade diante do desafio que representa a inclusão escolar, considerando as transformações paradigmáticas que envolvem este processo (PELOSI, 2006).

$\mathrm{Na}$ literatura internacional foram encontrados estudos que apresentam os movimentos de transformação e adaptação da formação e atuação do terapeuta ocupacional no processo de inclusão escolar desde o início da década de 1990 (NIEHUES, et al., 1991; STORCH; ESKOW, 1995; CASE-SMITH; CABLE, 1996; CLARK; MILLER, 1996; CASE-SMITH et al., 2001; PRIGG, 2002; WHALEN, 2003; WEINTRAUB; KOVSHI, 2004; MU et al., 2007, entre outros). Alguns destes trabalhos analisam tanto a realidade das intervenções quanto as tendências das práticas dos terapeutas ocupacionais e a partir dos resultados identificam diferentes “modelos de atuação" na inclusão escolar. Nesse sentido, possibilitam a identificação das mudanças ocorridas nos modos de atuar dos profissionais em função das transformações científicas e sociais, e mostram o que, na prática da Terapia Ocupacional, está sendo mantido e o que está sendo renovado e criado para determinados contextos como o da escola inclusiva.

No Brasil, identificam-se, na produção teórica da área, reflexões acerca da participação do terapeuta ocupacional no processo de inclusão escolar (GHIRARDI, 2000; BARTALOTTI; DE CARLO, 2001; ROCHA et al., 2001; ROCHA; ZULIAN, 2003; JURDI et al., 2004; CRUZ; DIMOV, 2005; PELOSI, 2006; ROCHA, 2007; TOYODA, 2007; LOURENÇO; CID, 2010). Observa-se, no entanto, a carência de estudos mais amplos abordando ações desenvolvidas no cotidiano deste processo. Os trabalhos consultados caracterizam-se principalmente pela reflexão sobre o papel da Terapia Ocupacional na educação inclusiva, pela apresentação de experiências e discussão das possíveis ações e contribuições da profissão neste contexto.

Acredita-se que as práticas cotidianas dos terapeutas ocupacionais brasileiros, assim como em outros países, indicam transformações e tendências a partir de suas ações na inclusão escolar. Identificar essas práticas e analisá-las

\footnotetext{
(1) Foi definido pela Portaria 2.344 de 3 de novembro de 2010 da Secretaria de Direitos Humanos da Presidência da República, a atualização da nomenclatura "pessoa portadora de deficiência” para o termo “pessoa com deficiência”. Entretanto, o presente estudo também usa "crianças com necessidades especiais" por ser considerado um termo adequado e utilizado em documentos e publicações relevantes e recentes da área.
} 
numa direção sistemática pode contribuir para a produção de conhecimento na área e para o desenvolvimento da profissão.

Neste sentido, foi desenvolvido no período de 2008 a 2009 um estudo com os terapeutas ocupacionais do estado de São Paulo que desenvolvem práticas relacionadas com o processo de inclusão escolar de crianças com necessidades educacionais especiais. O principal objetivo da pesquisa foi identificar e caracterizar, sob a ótica destes profissionais, as ações e realidade das práticas desenvolvidas no contexto da educação inclusiva.

\section{PROCESSO METODOLÓGICO}

Foi realizada uma pesquisa de levantamento, aprovada pelo Comitê de Ética da Universidade Federal de São Carlos (UFSCar) - CAAE 0083.0.135.000-07 de 12/03/2008.

O estudo contou com a participação de 127 terapeutas ocupacionais do estado de São Paulo, que desenvolvem ou já desenvolveram ações voltadas para a inclusão escolar nos dois últimos anos que antecederam a coleta de dados (coleta realizada de junho a agosto de 2008).

Havia o reconhecimento público de que estes profissionais atuavam no processo de inclusão escolar, mas não foram encontrados dados publicados sobre quantos eram, onde estavam inseridos e de que forma. Deste modo, para identificar os terapeutas ocupacionais envolvidos no processo de inclusão escolar e conhecer sua atuação e a diversidade de suas práticas foi necessário entrar em contato todos os associados ao Conselho Regional de Fisioterapia e Terapia Ocupacional do Estado de São Paulo - Crefito-3.

Para a coleta de dados três estratégias foram adotadas: 1) contato com os profissionais e coleta de dados por correio eletrônico (e-mail); 2) coleta de dados por endereço na internet (site); 3) contato com os profissionais sem endereço eletrônico válido por correio tradicional.

$\mathrm{Na}$ primeira fase da pesquisa, foram enviadas pelo Crefito-3 mensagens eletrônicas a todos os terapeutas ocupacionais associados com e-mail válido até a data de envio ( $n=2.861$ ), e remetidas, pelo pesquisador, cartas por correio postal ao restante dos profissionais $(\mathrm{n}=806)^{(2)}$.

A todos os terapeutas ocupacionais foi solicitado que respondessem "sim" ou "não" à seguinte questão:
"Você realiza ou já realizou em sua atuação profissional como terapeuta ocupacional, atividade(s) relacionada(s) ao processo de inclusão escolar de crianças com necessidades educacionais especiais?”.

Para todos os que responderam "sim", foram enviados os questionários contendo 26 questões (fechadas e abertas) que pretendiam identificar o participante e caracterizar seu envolvimento com a inclusão escolar e suas perspectivas em relação a esse processo. Os participantes poderiam responder às questões através do questionário anexado ao e-mail ou através de um link de acesso via internet, criado especialmente para essa finalidade.

Os dados coletados a partir de questões fechadas e abertas foram submetidos a Análises Exploratórias Descritivas, Análise de Conteúdo e Testes de Correlação. Parte dos resultados será apresentada e discutida a seguir.

\section{RESULTADOS E DISCUSSÃO}

\section{A relação dos terapeutas ocupacionais com a inclusão escolar}

O número de profissionais localizados no estudo ( $n=299$ ), envolvidos de alguma forma com o processo de inclusão escolar, representou $8,7 \%$ do total de terapeutas ocupacionais atuantes no estado de São Paulo e, portanto, associados ao Crefito-3. Destes, 47,5\% não responderam o questionário ou não responderam satisfatoriamente e desta forma obteve-se uma amostra de 127 participantes, analisada estatisticamente como representativa da população estudada.

Os participantes foram, principalmente, mulheres ( $\mathrm{n}=122)$ na faixa etária de 20 a 45 anos ( $\mathrm{n}=101)$ que, quando atuam na inclusão escolar, acompanham crianças de todas as idades, com destaque para a faixa etária de 6 a 10 anos $(\mathrm{n}=117)$.

Foi identificado o acompanhamento de crianças com necessidades educacionais especiais relacionadas a diferentes deficiências, com destaque de maior frequência para as deficiências intelectuais ( $\mathrm{n}=104)$ e físicas $(\mathrm{n}=89)$, e menor para deficiências auditivas $(n=30)$ e superdotação $(n=14)$.

O estudo revelou que esses terapeutas ocupacionais realizam práticas relacionadas ao processo de inclusão escolar principalmente em ambientes clínicos e na

\footnotetext{
(2) Foram considerados para a participação na pesquisa todos os terapeutas ocupacionais associados ao Crefito - 3 no dia 13/06/2008. Em respeito ao sigilo, os e-mails foram enviados pelo Conselho e os dados postais dos associados informados diretamente ao correio sem o conhecimento do pesquisador.
} 
escola regular, como pode ser observado nos resultados

apresentados nas Tabelas 1 e 2.

Tabela 1. Relação do terapeuta ocupacional com o processo de inclusão escolar

\begin{tabular}{l|c|c}
\hline De que forma sua atuação se relaciona com o processo de inclusão escolar? & $\begin{array}{c}\text { Porcentagem } \\
\text { (\%) }\end{array}$ & $\begin{array}{c}\text { Frequência } \\
\text { (n) }\end{array}$ \\
\hline $\begin{array}{l}\text { A partir do atendimento em ambiente clínico (consultório, instituição) de crianças em } \\
\text { processo de inclusão escolar }\end{array}$ & $85 \%$ & 108 \\
\hline Orientações esporádicas a escolas regulares & $43,5 \%$ & 68 \\
\hline Participação em equipe de apoio em escolas inclusivas regulares & $48,8 \%$ & 62 \\
\hline A partir da atuação em escola especial & $40,2 \%$ & 51 \\
\hline A partir da pesquisa na área da inclusão escolar & $27,6 \%$ & 35 \\
\hline Contratação para atendimento especializado em Terapia Ocupacional em escola regular & $18,1 \%$ & 23 \\
\hline Outras & $21,3 \%$ & 27 \\
\hline
\end{tabular}

N = 127 (sendo $\mathrm{N}$ o número total de participantes que responderam a questão)

Tabela 2. Principais ações realizadas pelos terapeutas ocupacionais no processo de inclusão escolar

\begin{tabular}{|c|c|c|}
\hline Ações realizadas pelos terapeutas ocupacionais & $\%$ & n \\
\hline Orientação à família em relação à escola e à inclusão & $95,3 \%$ & 121 \\
\hline Orientações gerais ao professor na escola regular & $89,8 \%$ & 114 \\
\hline $\begin{array}{l}\text { Atividades em ambiente clínico/instituição para o desenvolvimento de habilidades cognitivo-motoras } \\
\text { da criança em processo de inclusão escolar. }\end{array}$ & $82,7 \%$ & 105 \\
\hline Orientações gerais à escola regular & $82,7 \%$ & 105 \\
\hline Orientações específicas sobre uso de tecnologia assistiva e/ou mobiliário & $78,7 \%$ & 100 \\
\hline Treinamento de AVDs na clínica/instituição e orientação para essas atividades na escola regular & $72,4 \%$ & 92 \\
\hline
\end{tabular}

$\mathrm{N}=127$

Estes achados se assemelham aos de estudos internacionais (CASE-SMITH; CABLE, 1996; WEINTRAUB; KOVSHI, 2004, entre outros) que identificaram a atuação do terapeuta ocupacional no processo de inclusão escolar especialmente na clínica e na escola comum.

No questionário, o ambiente clínico foi especificado aos participantes como consultórios e instituições e as atividades para o desenvolvimento de habilidades cognitivomotoras da criança em processo de inclusão escolar são realizadas nestes locais pela maioria dos participantes. Tais resultados apoiam as considerações de Bartalotti e De Carlo (2001) que discutem que a atuação da Terapia Ocupacional está tradicionalmente relacionada à reabilitação, com práticas consolidadas voltadas para a minimização de sequelas e dificuldades e para a promoção do desempenho funcional.

Essas práticas são importantes para a clientela que dela se beneficia, no entanto, a literatura indica que ao se relacionar com o processo de inclusão escolar, o terapeuta ocupacional não deve restringir suas ações ao ambiente clínico, estas devem ser contextualizadas com as práticas educacionais que envolvem o cotidiano escolar das crianças (BARTALOTTI; DE CARLO, 2001), assim como foi identificado nas práticas dos terapeutas ocupacionais deste estudo.

Observou-se que, embora a grande maioria dos participantes se relacione com a inclusão escolar a partir dos atendimentos em ambiente clínico, as ações dos terapeutas ocupacionais estão voltadas para e realizadas na escola regular. Tal observação é relevante, pois sugere um movimento na direção da complementação do atendimento 
clínico através da relação com o cotidiano educacional.

Rocha et al. (2001) discutiram a inclusão escolar de crianças com necessidades educacionais especiais e apontaram que, no Brasil, a experiência da Terapia Ocupacional neste contexto se encontrava restrita ao acompanhamento de alunos em escolas especiais ou em instituições relacionadas ao atendimento clínico, que preparavam os indivíduos para uma inserção social ainda pouco voltada para a escola comum. Os resultados do presente estudo apontam um novo direcionamento das práticas e, deste modo, indicam novas formas de relação dos terapeutas ocupacionais com a educação inclusiva, uma vez que foi identificado um importante enfoque destes profissionais na inclusão escolar de seus clientes e a própria atuação na escola regular.

\section{As ações}

Os terapeutas ocupacionais participantes apontaram como suas principais ações realizadas para favorecer a inclusão escolar (Tabela 2): a) a orientação à família em relação à escola e à inclusão; b) orientação aos professores e à escola em geral; c) atividades realizadas em ambiente clínico/instituição para o desenvolvimento de habilidades cognitivo-motoras; d) orientações à escola para adequação/ adaptação de espaços físicos e recursos materiais e e) treinamento de AVDs (Atividades de Vida Diária) na clínica/instituição e orientação para essas atividades na escola regular.

Foram identificadas ações relacionadas a três contextos principais: família, ambiente clínico e escola. No entanto, é importante destacar o resultado obtido a partir da última questão do questionário (aberta e optativa), em que foi solicitado aos participantes que descrevessem um ou mais casos vivenciados no processo de inclusão escolar que considerassem como "bem sucedidos". Os resultados, esboçados na Tabela 3 a seguir, apontaram que os participantes relacionam o sucesso das intervenções principalmente às ações realizadas pontualmente na escola regular.

Tabela 3. Intervenções e ações bem sucedidas apontadas nos depoimentos (Questão optativa - Análise de Conteúdo)

\begin{tabular}{l|c|c}
\hline Intervenções e ações bem sucedidas relacionadas ao processo de inclusão escolar & \% & n \\
\hline $\begin{array}{l}\text { Inclusão ou inserção na escola regular: preparação e/ou capacitação da escola e dos professores para } \\
\text { receber alunos com necessidades especiais e efetivação da inclusão ou a inserção na escola regular } \\
\text { (creches, escolas de ensino infantil e fundamental e universidades) }\end{array}$ & $52,3 \%$ & 33 \\
\hline $\begin{array}{l}\text { Maior participação e autonomia nas atividades escolares e socialização: transformações de comporta- } \\
\text { mento e atitude tanto dos alunos acompanhados no ambiente escolar quanto dos profissionais da escola } \\
\text { e dos outros alunos }\end{array}$ & $38,1 \%$ & 24 \\
\hline $\begin{array}{l}\text { Resultados positivos nas adequações e adaptações em sala de aula regular e no desenvolvimento peda- } \\
\text { gógico: implementação da tecnologia assistiva e da comunicação alternativa e adaptação de atividades } \\
\text { pedagógicas; }\end{array}$ & $31,7 \%$ & 20 \\
\hline $\begin{array}{l}\text { Sensibilização, conscientização e discussão com familiares, alunos e profissionais envolvidos com } \\
\text { educação especial e com o processo de inclusão escolar }\end{array}$ & $12,6 \%$ & 8 \\
\hline $\begin{array}{l}\text { Desenvolvimento neuropsicomotor ou a “evolução clínica” das crianças em processo de inclusão esco- } \\
\text { lar }\end{array}$ & $11,1 \%$ & 7 \\
\hline
\end{tabular}

$\mathrm{N}=63$

Este resultado sugere que os participantes valorizam as ações realizadas no contexto educacional. De forma geral, a análise dos dados possibilitou identificar, que os terapeutas reconhecem a existência de forte relação entre a intervenção na escola e a efetiva contribuição para o processo de inclusão escolar.

$\mathrm{Na}$ intervenção na escola regular, as principais ações descritas pelos terapeutas ocupacionais foram: a orientação dos professores; a orientação da escola em geral e a orientação para adequação/adaptação de espaços físicos e recursos materiais, como pode ser observado a partir das informações apresentadas nas Tabelas 2 e 3.

Quando voltados à orientação dos professores, os participantes destacaram principalmente a conscientização sobre as potencialidades das crianças; a capacitação para utilização de tecnologia assistiva e a orientação sobre adaptações em atividades/dinâmicas em sala de aula. Foi apontado ainda a realização de intervenções realizadas em 
conjunto com o professor de forma colaborativa, conforme pode ser observado depoimento a seguir:

(...) O trabalho com a escola foi pensar juntas estratégias que favorecessem o potencial criativo do W. e também o potencial das professoras, que elas achavam insuficiente para dar conta do insucesso escolar de W., para propiciar o aprendizado, que tanto ele quanto elas desejavam (...)

Tais resultados reforçam a premissa de que o terapeuta ocupacional entende o fazer do professor como mais um foco de sua atuação no processo de inclusão escolar (BARTALOTTI, 2007) e apresenta possibilidades significativas de contribuir com a capacitação deste profissional para atuar na perspectiva inclusiva (ZULIAN et al., 2004).

Esta discussão chama a atenção para a importância da participação da Terapia Ocupacional na preparação e fortalecimento da equipe escolar, uma vez admitida a necessidade de sensibilização e treinamento dos recursos humanos da escola para efetivamente prepará-la para incluir alunos com necessidades educacionais especiais (SASSAKI, 2002).

Outra ação realizada na escola e fortemente identificada foi a orientação sobre tecnologia assistiva e adequação/adaptação de espaços físicos e recursos materiais.

Destaca-se que nas questões fechadas do questionário, a opção dada aos participantes foi apresentada de forma limitada ao se referir apenas à "orientação sobre tecnologia assistiva e mobiliário”, o que não impediu que os profissionais apontassem outras ações relacionadas a este aspecto nas questões e itens abertos do instrumento, especialmente nos relatos de casos bem sucedidos, tais como: adaptações de materiais escolares para a realização de atividades em sala de aula; implementação de computador com teclado adaptado e treinamento do aluno e professor para a utilização; prescrição e confecção de órteses; adequação de mobiliários e utilização de equipamentos específicos para melhorar a acessibilidade; implementação de comunicação alternativa, entre outras.

Tais resultados confirmam reflexões de autores que discutem o envolvimento do terapeuta ocupacional com a tecnologia assistiva como uma das possíveis ações no processo de inclusão escolar (BARTALOTTI; DE CARLO, 2001; ROCHA et al., 2001; PELOSI, 2006; ROCHA, 2007; TOYODA et al., 2007).

\section{Obstáculos, desafios e perspectivas}

O estudo procurou identificar, na visão dos terapeutas ocupacionais participantes, os principais obstáculos e desafios para a atuação na inclusão escolar. Os resultados apontaram a falta de preparação da escola em geral e dos professores para receber alunos com necessidades especiais em suas salas de aula como fatores importantes, como pode ser observado na Tabela 4.

Tabela 4. Obstáculos e desafios na realização do trabalho do terapeuta ocupacional na inclusão escolar

\begin{tabular}{l|c|c}
\hline Obstáculos e desafios & \multicolumn{2}{c}{ n } \\
\hline $\begin{array}{l}\text { Falta de preparação dos professores para receber alunos com necessidades especiais em suas salas de } \\
\text { aula regulares }\end{array}$ & $91,1 \%$ & 112 \\
\hline Falta de preparação da equipe da escola regular para o processo de inclusão escolar & $89,4 \%$ & 110 \\
\hline Falta de recursos materiais (material para adaptação, dentre outros) na escola regular & $65 \%$ & 80 \\
\hline Limitações estruturais da escola regular para a atuação do terapeuta ocupacional na escola & $61 \%$ & 75 \\
\hline Limitação de tempo para a atuação do terapeuta ocupacional na escola regular & $51,2 \%$ & 63 \\
\hline $\begin{array}{l}\text { Dificuldade da escola regular em receber orientação de terapeutas ocupacionais que atendem na } \\
\text { clínica crianças incluídas }\end{array}$ & $37,4 \%$ & 46 \\
\hline Falta de cooperação dos familiares no trabalho da Terapia Ocupacional & $26,8 \%$ & 33 \\
\hline Falta de cooperação do professor no trabalho da Terapia Ocupacional & $26 \%$ & 32 \\
\hline Outros & $22,8 \%$ & 28 \\
\hline N = 123 & \multirow{2}{*}{$3 \%$}
\end{tabular}


Em relação à falta de preparação dos professores, é importante destacar que os participantes diferenciaram "preparação" de "cooperação", ou seja, não apontaram a falta de cooperação dos professores como um obstáculo importante, mas destacaram sua falta de preparação/ capacitação. Diferente do resultado encontrado por Prigg (2002) que verificou que para os terapeutas ocupacionais australianos envolvidos com a inclusão escolar de crianças no período de iniciação na escola regular uma das principais dificuldades para atuar se relacionava à falta de participação e cooperação dos professores e da equipe da escola.

Quanto aos profissionais do presente estudo, acreditase que este fato revelou a percepção da diferença entre "não estar preparado" e "não querer cooperar". Tais aspectos são muitas vezes confundidos no contexto da escola, à medida que o despreparo do professor, ator essencial no processo de inclusão escolar, potencializa sentimentos de impotência e medo diante do desconhecido, de um desafio que não se sabe como enfrentar. Para Rocha et al. (2003) os professores sentem-se solitários e despotencializados em uma proposta que necessita de parceiros e apoio e, muitas vezes, por desconhecerem questões básicas sobre os alunos com necessidades educacionais especiais, sentem-se impotentes em sua ação como educador.

Outros aspectos que aparecem como desafios para a atuação dos terapeutas ocupacionais na inclusão escolar dizem respeito à preparação da equipe da escola em geral e a falta de estrutura e de recursos materiais.

A questão do despreparo da equipe escolar e da escola como um todo, debatido por vários estudiosos do tema, parece remeter a uma discussão anterior que indaga sobre como a escola exerce seu papel na construção do respeito à diversidade. E, nesta direção, as propostas da educação inclusiva muitas vezes questionam "alguns dos fundamentos mais arraigados da escola tradicional: o caráter seletivo, a homogeneidade dos seus métodos de ensino e o fato de não ser sensível ao que os alunos são e querem” (RODRIGUES, 2006, p.317).

Acredita-se que é preciso considerar a inclusão escolar, bem como a preparação dos professores e da própria escola, como um processo em construção. As medidas legislativas no Brasil nos últimos anos foram um passo importante para a inclusão escolar, mas esta será (e está sendo) efetivamente construída a partir das práticas e das reflexões que surgem delas.

A escola preparada para a inclusão será a escola que emergirá dos esforços e da coragem dos atores diretamente envolvidos, ou seja, de toda a comunidade (RODRIGUES, 2006) e nessa perspectiva, alguns resultados do presente estudo apontam para a participação do terapeuta ocupacional neste movimento.

Em questão aberta, foi solicitado aos terapeutas ocupacionais o apontamento de ações que deveriam ser realizadas pela Terapia Ocupacional no processo de inclusão escolar, mas que ainda não ocorrem ou ocorrem raramente. Os principais apontamentos foram:

$\checkmark$ Inserção no cotidiano das escolas, nas salas de aula e nas equipes do ensino regular;

$\checkmark$ Adaptação de ambientes, de mobiliários e de atividades didáticas e lúdicas, bem como criação de estratégias e orientação para a utilização dos mesmos; e planejamento, avaliação e implementação de recursos de tecnologia assistiva e comunicação alternativa;

$\checkmark$ Orientação, apoio e trabalho em conjunto com professores e/ou formação/capacitação do professor;

$\checkmark$ Atuação na comunidade (conscientização e incentivo para participação no processo de inclusão escolar).

Destaca-se que os principais apontamentos dizem respeito a ações que foram identificadas nas outras respostas dos participantes, ou seja, ações que já fazem parte das práticas destes profissionais. Assim, verifica-se que os participantes do estudo indicaram que alguns aspectos já presentes em sua atuação merecem ser ampliados; este resultado sugere a reflexão de uma prática que pode estar indicando ações positivas e efetivas para educação inclusiva e por isso são apontadas.

Ainda sob essa perspectiva, é interessante constatar que três das quatro principais ações que devem ser ampliadas, na visão dos participantes, vão diretamente ao encontro dos maiores obstáculos encontrados e auxiliam na preparação da escola e dos professores e na construção do processo de inclusão escolar.

Nesse mesmo sentido, foi solicitado aos participantes sugestões para as políticas públicas; cotidiano escolar e pesquisas/formação do terapeuta ocupacional na área da educação inclusiva que poderiam contribuir com a efetivação da inclusão escolar. Observou-se que os resultados também se relacionam com os principais obstáculos e desafios identificados no estudo.

Em relação às políticas públicas, os participantes acreditam que deve haver maior investimento: a) na contratação e capacitação dos profissionais das escolas regulares, especialmente do professor; b) na preparação estrutural das escolas e c) na contratação de profissionais técnicos para equipes de apoio, com destaque para o terapeuta ocupacional.

Os participantes demonstram reflexão sobre a 
realidade do processo de inclusão escolar e identificam a participação da Terapia Ocupacional como uma alternativa eficiente de apoio.

Estes resultados chamam a atenção para o fato de que, embora a legislação aponte para a tendência e a prioridade política de capacitar os professores e a escola para receber os alunos em com necessidades educacionais especiais (LDB/BRASIL, 1996; PNE/BRASIL, 2001; PNEEPEI/BRASIL, 2008), políticas públicas e programas mais enfáticos nesta direção precisam ainda ser elaboradas e concretizadas, considerando o terapeuta ocupacional como um profissional colaborador deste processo.

Acredita-se ser importante o investimento na atuação de terapeutas ocupacionais no processo de inclusão escolar, à medida que, com base nos dados aqui apresentados, esses profissionais já se encontram envolvidos com a preparação das escolas e dos professores e identificam suas ações como fator contribuinte importante neste aspecto.

\section{CONSIDERAÇÕES FINAIS}

$$
\text { O presente estudo identificou e apresentou }
$$

características representativas da atuação de terapeutas ocupacionais do estado de São Paulo no processo de inclusão escolar. A partir dos resultados obtidos, confirmase o potencial colaborativo deste profissional na educação inclusiva e sugere-se que estudos e programas sejam desenvolvidos por representantes da área e pelas instâncias públicas a fim de explorar e utilizar os conhecimentos da Terapia Ocupacional na efetivação da inclusão escolar no Brasil.

Destaca-se ainda os resultados positivos da utilização de recursos da "tecnologia da informação e comunicação" - e-mail e site, como estratégia para a coleta de dados neste estudo. Obteve-se uma boa aceitação dos participantes em relação ao método e uma amostra representativa, a partir de um processo mais ágil e econômico quando comparado aos métodos tradicionais, como utilização de correio postal, por exemplo.

Por fim, agradecemos especialmente aos terapeutas ocupacionais participantes da pesquisa que contribuíram com compromisso e afeto para a concretização deste estudo e ao Crefito-3 por sua colaboração fundamental.

CARDOSO, P. T.; MATSUKURA, T. S. Practice and perspectives of school-based occupational therapy. Rev. Ter. Ocup. Univ. São Paulo, v. 23, n. 1, p. 7-15, jan./abr. 2012.

\begin{abstract}
The objective of the present study was to identify and characterize the school-based Occupational Therapy actions towards children with educational special needs in the state of Sao Paulo. A statistic research in which 127 occupational therapists participated has been carried out. The data collected by means of a questionnaire was submitted to quantitative and qualitative analysis. The main findings indicate that school-based occupational therapists: a) relate to this process mainly through appointments in clinical settings; b) work in regular schools; c) have family and other professionals' participation in their practice; d) identify as the greatest obstacles for their practice the school and teachers' lack of preparation; e) point out the importance and need of a broader insertion of Occupational Therapy in regular schools. The study identified representative characteristics in the performance of school-based occupational therapists in Sao Paulo. It also pointed out indicators for future research.
\end{abstract}

KEYWORDS: Disabled children/education; Education, special/trends; Education, special/human resources; Occupational therapy.

\title{
REFERÊNCIAS
}

BARTALOTTI, C. Deficiência mental. In: CAVALCANTI, A.; GALVÃO, C. (Org.). Terapia 0cupacional: fundamentação \& prática. Rio de Janeiro: Guanabara Koogan, 2007. p. 295-298.

BARTALOTTI, C.; DE CARLO, M. A terapia ocupacional e os processos socioeducacionais. In: DE CARLO, M.; BARTALOTTI, C. (Orgs.). Terapia ocupacional no Brasil: fundamentos e perspectivas. São Paulo: Plexus, 2001. p. 99-116.
BRASIL. Ministério da Educação e da Cultura. Lei de Diretrizes e Bases da Educação Nacional. Lei no 9.394, de 20 de dezembro de 1996. Brasília, DF, 1996. Disponível em: <http://portal.mec. gov.br/arquivos/pdf/ldb.pdf $>$. Acesso em: 27.05.2011.

BRASIL. Ministério da Educação e da Cultura. Plano Nacional de Educação Decretado pelo Congresso Nacional. Brasília, DF, 2001. Disponível em: <http://portal.mec.gov.br/arquivos/pdf/pne. 
pdf>. Acesso em: 27.05.2011.

BRASIL. Ministério da Educação e da Cultura. Secretaria de Educação Especial. Política Nacional de Educação Especial na Perspectiva da Educação Inclusiva. Brasília, DF, 2008. Disponível em: http://portal.mec.gov.br/seesp/arquivos/pdf/politica.pdf. Acesso em: 27.05.2011.

CASE-SMITH, J.; CABLE, J. Perceptions of occupational therapists regarding service delivery models in school-based practice. Occup. Ther. J. Res., v. 16, n. 1, p. 23-44, 1996.

CASE-SMITH, J.; ROGERS, J.; JOHNSON, J. School-based occupational therapy. In: CASE-SMITH, J. (Org.). Occupational therapy for children. Ohio, USA, 2001. p. 757-779.

CLARK, G.; MILLER, L. Providing effective occupational therapy services: data-based decision making in school-based practice. Am. J. Occup. Ther., v. 50, n. 9, p. 701-708, 1996.

CRUZ, D.; DIMOV, T. Uma discussão com docentes acerca das contribuições da Terapia Ocupacional na educação especial. Temas Sobre Desenvol., v. 14, n. 80-81, p.40-46, 2005.

GHIRARDI, M. Educação inclusiva, processos psicológicos e a Terapia Ocupacional. Rev. Ter. Ocup. Univ. São Paulo, v. 11, n. 1, p. 13-6, 2000.

JURDI, A.; BRUNELLO, M.; HONDA, M. Terapia Ocupacional e propostas de intervenção na rede pública de ensino. Rev. Ter. Ocup. Univ. São Paulo, v.15, n. 1, p.26-32, 2004.

LOURENÇO, G.; CID, M. Possibilidades de ação do terapeuta ocupacional na educação infantil: congruência com a proposta da educação inclusiva. Cad. Ter. Ocup. Ufscar, São Carlos, v.18, n. 2, p.169-179, 2010.

MENDES, E. Perspectivas para a construção da escola inclusiva no Brasil. In: PALHARES, M.; MARINS, S. (Orgs.). Escola inclusive. São Carlos, SP: EdUFScar, 2002. p. 61-85.

MU, K.; FRANCK, L.; KONZ, C. Attitudes of entry level occupational therapy doctoral students towards inclusion for students with disabilities. Austr. Occup. Ther. J., v.54, p. 66-73, 2007.

NIEHUES, A.; et al. Making a difference: occupational therapy in the public schools. Occup. Ther. J. Res., v. 11, n. 4, p. 195-212, 1991.

PELOSI, M. Por uma escola que ensine e não apenas escolha recursos e estratégias para a inclusão escolar. In: MANZINI, E. (Org). Inclusão e acessibilidade. Marilia: ABPEE, 2006. p. 121-132.

PRIGG, A. Experiences and perceived roles of occupational therapists working with children with special learning needs during transition to school: a pilot study. Austr. Occup. Ther. J., v. 49, p.100-111, 2002.

ROCHA, E. A terapia ocupacional e as ações na educação: aprofundando interfaces. Rev. Ter. Ocup. Univ. São Paulo, v. 18, n. 3, p. 122-127, 2007

ROCHA, E.; CASTIGLIONI, M.; VIEIRA, R. A inclusão da criança com deficiência na escola comum: reflexões sobre o papel da Terapia Ocupacional. Rev. Ter. Ocup. Univ. São Paulo, v. 12, n. 1/3, p. 8-14, 2001.

ROCHA, E.; LUIZ, A.; ZULIAN, M. Reflexões sobre as possíveis contribuições da Terapia Ocupacional nos processos de inclusão escolar. Rev. Ter. Ocup. Univ. São Paulo, v. 14, n. 2, p. 72-8, 2003.

RODRIGUES, D. Dez ideias (mal) feitas sobre a educação inclusiva. In: RODRIGUES, D. (Org.). Inclusão e educação: doze olhares sobre a educação inclusiva. São Paulo: Summus, 2006. p. 299-318.

SASSAKI, R. Inclusão: construindo uma sociedade para todos. 4a. ed. Rio de Janeiro: WVA, 2002.

SOARES, L. Terapia ocupacional: lógica do capital ou do trabalho? Retrospectiva histórica da profissão no estado brasileiro de 1950 a 1980. São Paulo: Hucitec, 1991.

STORCH, B.; ESKOW, K. Theory application by school based occupational therapists. Am. J. Occup. Ther., v. 50, n. 8, p. 662668, 1995.

TOYODA, C.; et al. O contexto multidisciplinar da prática da Terapia Ocupacional frente ao paradigma da inclusão escolar. Cad. Ter. Ocup. Ufscar, São Carlos, v. 15, n. 2, p. 121-130, 2007.

WEINTRAUB, N.; KOVSHI, M. Changing practice patterns of school-based occupational therapistis in Israel. Occup. Ther. Int., v. 11, n. 1, p. 40-51, 2004.

ZULIAN, M.; et al. A terapia ocupacional em processos interdisciplinares da educação especial. In: OMOTE, S. (Org). Inclusão: intenção e realidade. Marília: Fundepe, 2004. p. 201211.

Recebido para publicação: 20/09/2011

Aceito para publicação: 22/03/2012 